\title{
Long Term Follow-up of Cases of Uveal Effusion Syndrome Treated with Partial Thickness Sclerectomies
}

\author{
Muhammad Tariq Khan, Sidrah Riaz, Haroon Tayyab, Abdul Majeed Malik
}

Pak J Ophthalmol 2019, Vol. 35, No. 4

See end of article for

authors affiliations

Correspondence to:

Dr. Muhammad Tariq Khan Ophthalmology Department Akhtar Saeed Trust Hospital, Email: stariq69@hotmail.com
Purpose: To report the effect of partial thickness sclerectomy in patients with idiopathic uveal effusion syndrome.

Study Design: Interventional case series.

Place and Duration of Study: Multi-center study at LRBT eye hospital, Medicare hospital and Mayo hospital, Lahore from January 2010 to August2015.

Material and Methods: Total six eyes of four patients (two males and two females) with bilateral idiopathic uveal syndrome were included in the study. The diagnosis was clinically confirmed on B scan ultrasonography, which confirmed relatively short axial length, exudative retinal detachment and scleral thickening. The surgical procedure included 360 degrees peritomy, followed by partial thickness sclerotomies measuring $4 \times 6 \mathrm{~mm}$ placed in between the recti muscles.

Results: All six (6) patients showed clinical improvement in visual acuity. Preoperative visual acuity was perception of light (PL) in all cases which improved to $6 / 36-6 / 24$ at 12 weeks follow up. On fundoscopy and B scan ultrasound there was resolution of exudative retinal detachment after partial thickness sclerectomies in all cases. The intraocular pressure was high $(26 \mathrm{~mm} \mathrm{Hg})$ in only one case which improved to $18 \mathrm{~mm} \mathrm{Hg}$ at 12 weeks follow-up. All other cases had IOP within normal limits pre and post operatively.

Conclusion: Partial thickness sclerectomy is an effective treatment option for patients of uveal effusion syndrome not responding to medical treatment.

Key Words: Uveal effusion syndrome, Sclerectomy, exudative retinal detachment (RD), intraocular pressure (IOP),
I t was 1958 when von Graefe ${ }^{1}$, Verhoeff and Waite ${ }^{2}$ defined spontaneous serous detachment of choroid. In 1963, Schepens and Brockhurst ${ }^{3}$ coined the term "uveal effusion". They reported spontaneous exudative detachment of choroid and ciliary body. In 1982, Gass and Jallow introduced the term "idiopathic uveal effusion syndrome" (UES) to describe idiopathic serous detachment of choroid, ciliary body and retina ${ }^{4}$. It is a rare ocular disorder affecting predominantly healthy young males and involvement is commonly bilateral. Albumin in the choroidal capillaries draws fluid into blood vessels and maintains relative dehydration of the
Suprachoroidal space. Fenestrated capillaries of the choroid allow albumin to escape into the extra vascular space. To maintain the colloid osmotic gradient, albumin leaves the choroid across the sclera and this trans-scleral protein flow is facilitated by intraocular pressure. This equilibrium is disturbed by neoplastic and inflammatory diseases leading to accumulation of protein, mostly albumin, in the extra vascular space of the choroid. The higher colloid osmotic pressure reduces movement of fluid from the Suprachoroidal space into the choroidal capillaries leading to accumulation in Suprachoroidal fluid and serous ciliochoroidal effusion. Scleral thickness, scleral 
composition and the number of scleral emissary channels obstructs diffusion of protein out of eye, reduces aqueous flow through uveo-scleral outflow and vortex vein compression in nanophthalmic eyes leading to osmotic pressure gradient, which causes fluid accumulation in the Subretinal space, resulting in secondary serous retinal detachment. It is a diagnosis of exclusion. The clinical features are nonrhegmatogenous retinal detachment (RD) with marked shifting fluid. Retinal detachment often begins inferiorly like other exudative detachments. Fluid accumulation is always more anteriorly because anterior fibers attaching choroid to sclera are long and tangentially oriented whereas posterior fibers are short $^{5}$. Other features are dilated episcleral vessels, blood in canal of schlemm, normal intraocular pressure (IOP), elevation of sub retinal fluid protein levels and elevated levels of cerebrospinal fluid (CCF) proteins. Striking changes may be evident at level of retinal pigment epithelium with so called "leopard spot" hyper pigmentation ${ }^{3}$. There is no evidence of intraocular inflammation except from few vitreous cells, which are present occasionally ${ }^{3}$. The natural history of disease is usually prolonged with remission and without treatment; patients may experience permanent loss of vision. The milder forms usually resolve but visual prognosis for eyes presenting with bullous retinal detachment is poor ${ }^{6}$.

Jackson et al revealed that UES can be associated with reduced trans-scleral albumin permeability. ${ }^{7}$ It is divided into 3 types: Type 1 is nanophthalmic eyes and high hyperopic patients. Type 2 is nonnanophthalmic eyes with abnormal sclera, normal eyeball and small refractive errors. Type 3 is nonnanophthalmic eye with normal sclera. Nanophthalmic eyes plus presence of clinically detectable thickened and rigid sclera is a good predictor for histological abnormal sclera and provides good response to surgery ${ }^{8}$. Trelstad et al found that in uveal effusion syndrome, the sclera showed histo-chemical abnormalities: The bundles of collagen fibers in the sclera had a markedly irregular arrangement and varied in width. They also noted abnormal deposition of glycosaminoglycan among the collagen bundles. ${ }^{9}$ The diagnostic value of FFA and ICGA and OCT is limited in uveal effusion syndrome and serves mostly to exclude other etiologies. Spectraldomain optical coherence tomography may show focal thickening of the retinal pigment epithelium layer corresponding to the areas of leopard spots ${ }^{10}$.

It responds poorly to medical treatment like corticosteroids and anti metabolites ${ }^{4}$ and non-surgical treatment. Medical therapy has been described as a possible first step before surgical approach and includes topical prostaglandin analogs and/or oral carbonic anhydrase inhibitors ${ }^{11,12}$. Although Brockhurst described good surgical results with decompression of vortex veins, but limitations like difficulty to isolate the veins and vein rupture made this procedure complicated and so was not preferred ${ }^{13}$. Successful surgeries, with no risk/damage to vortex vein, like use of Express Shunt for choroidal drainage in UES has also been documented ${ }^{14}$.

Uyama et al. reported that a small sclerectomy under the scleral flap could be effective in both type 1 and type 2 UES because the abnormal sclera and increased resistance to the trans-scleral outflow of intraocular fluid are thought to be the main causes of these disorders ${ }^{1}$ The full-thickness drainage sclerectomy resulted in resolution of UES, including type 3 UES, and a subsequent improvement in visual acuity. The effect may come from indirect decompression of vortex veins by relaxing scleral tension ${ }^{15}$. Some reports have shown that $10 \mathrm{mg}$ dexamethasone given intravenous (IV) prior to surgery also improves the surgical outcome. A recent report showed that combining IV steroids with partial thickness sclerectomies yielded excellent anatomic results. The final visual gains were moderate in this report ${ }^{16}$.

Response to surgical options like scleral buckling and pars plana vitrectomy (PPV) is also not convincing. Successful retinal re-attachment of nonrhegmatogenous retinal detachment requires a scleral thinning procedure, including quadrantic partial thickness sclerectomies. The purpose of this case series was to study the effect of partial thickness sclerectomy in patients with idiopathic uveal effusion syndrome.

\section{MATERIALS AND METHODS}

Over the period of five years, six eyes of four patients ( 2 males and 2 females) with bilateral idiopathic uveal syndrome were included in study. The diagnosis was clinical. They had no evidence of posterior scleritis, orbital inflammatory disease, arteriovenous fistula, recent pan retinal photocoagulation, retinal surgery, recent ocular trauma, ocular neoplasm or drug reaction to sulfonamides or acetazolamide.

The surgical procedure included 360 degrees peritomy, followed by partial thickness sclerctomies measuring $4 \times 6 \mathrm{~mm}$ placed in between the recti 
muscles. The sclerectomies were performed in all quadrants with the anterior border just anterior to the insertion of the recti muscles. All patients were followed up for one week, three weeks and twelve weeks postoperatively.

\section{Case 1}

A young male presented with gradual decrease in vision (perception of light) for last 6 months. His intraocular pressure (IOP) was $10 \mathrm{mmHg}$ in both eyes. Quadrantic sclerectomies were done in both eyes with 1 week interval. Subretinal fluid (SRF) was drained in left eye but not in right eye. Hypotony was overcome in left eye with intravitreal $\mathrm{C}_{3} \mathrm{~F}_{8}$ injection after SRF drainage. He showed progressive vision improvement over 3 months and his exudative retinal detachment also improved over 12 weeks.

\section{Case 2}

A young female presented with gradual decrease in visual acuity for last 9 months. Her visual acuity was light perception (PL) both eyes and IOP was $8 \mathrm{mmHg}$ in right and $6 \mathrm{~mm} \mathrm{Hg}$ in left eye respectively. She was wearing hypermetropic glasses of +7 DS in both eyes. Her axial length was $19 \mathrm{~mm}$ in both eyes. Her right eye was operated with quadrantic sclerectomies, her vision improved in that eye. She was advised same surgical procedure for the left eye but she did not report back for left eye surgery after 2 follow up visits.

\section{Case 3}

A 28 years old young lady with bilateral pseudophakia, presented with decreased vision, (counting fingers OD and 6/60 OS). Her record showed IOL power was +33 diopters OD and +36 diopters OS. She was under-corrected per-operatively with a residual post operative refractive error of $+8 \mathrm{D}$ and $+10 \mathrm{D}$ glasses. Axial length was $18 \mathrm{~mm}$ in both eyes. IOP was recorded as $15 \mathrm{mmHg}$ on presentation. She had bilateral exudative retinal detachment on $\mathrm{B}$ scan. She underwent bilateral quadrantric sclerectomies under general anesthesia.

\section{Case 4}

A 26 years old male presented in OPD with gradual decrease in vision. His vision was 6/60 OD and 6/12 OS. IOP was $26 \mathrm{~mm} \mathrm{Hg}$ OD and $18 \mathrm{~mm} \mathrm{Hg}$ OS. Axial length was $18.14 \mathrm{~mm}$ OD and $18.03 \mathrm{~mm}$ OS. CD ratio was 0.2 in both eyes. Auto refractometer showed hypermetropia of $+18 \mathrm{D}$ and $+18.5 \mathrm{D}$. On B scan 360 degrees choroidal detachment was observed in right eye. Left eye was normal.

\section{RESULTS}

Six eyes of four patients were operated and quadrantic sclerectomies was performed in these. The results are shown in table 1.

Case 1 showed improvement in vision, which was faster in left eye than right eye, in which sclerectomy was accompanied by SRF drainage and intravitreal $\mathrm{C}_{3} \mathrm{~F}_{8}$ gas injection under general anesthesia. His vision improved from PL to 6/24 four weeks postoperatively in left eye and from PL to $6 / 24$ six weeks postoperatively in right eye without SRF drainage. His retina was attached in both eyes. He was examined on his last follow-up visit on September 2014 and he had visual acuity of $6 / 18$ with normal IOP in both eyes.

Case 2 was examined one and three weeks postoperatively after sclerectomy under general anesthesia. Her vision improved from PL to 6/36 with glasses in her right eye. Her retina was attached. She did not return for surgery of the second eye.

Case 3 was pseudophakic female who was operated for both eyes with an interval of 3 months. Quadrantic sclerectomies were done under general anesthesia. She showed gradual visual improvement over a period of 12 weeks. Her exudative retinal detachment also improved.

Case 4 was a hypermetropic male who underwent 2 sclerectomies in superotemporal and inferotemporal quadrant in right eye under local anesthesia and sedation. His vision improved and exudative detachment also improved on B scan.

Table 1: Pre and post-operative visual acuity and IOP of the patients.

\begin{tabular}{|c|c|c|c|c|c|c|c|c|}
\hline \multirow{3}{*}{ Sr. \# } & \multicolumn{2}{|c|}{ Pre-Operative } & \multicolumn{6}{|c|}{ Post-Operative } \\
\hline & \multirow[t]{2}{*}{ Visual Acuity } & \multirow{2}{*}{ IOP (mm Hg) } & \multicolumn{3}{|c|}{ Visual Acuity } & \multicolumn{3}{|c|}{ IOP (mm Hg) } \\
\hline & & & 1 Week & 3 Weeks & 12 Weeks & 1 Week & 3 Weeks & 12 Weeks \\
\hline Case 01 & Right eye PL+ve & 10 & $6 / 36$ & $6 / 24$ & $6 / 24$ & 10 & 10 & 10 \\
\hline Male & Left eye PL+ve & 10 & $6 / 36$ & $6 / 24$ & $6 / 24$ & 12 & 10 & 10 \\
\hline Case 02 & Right eye PL+ve & 8 & $6 / 36$ & $6 / 36$ & $6 / 36$ & 10 & 12 & 10 \\
\hline
\end{tabular}




\begin{tabular}{|c|c|c|c|c|c|c|c|c|}
\hline Female & Left eye PL +ve & 10 & surgery not done & - & - & - & - & - \\
\hline Case 03 & Right eye PL+ve & 8 & $6 / 36$ & $6 / 36$ & $6 / 36$ & 10 & 12 & 10 \\
\hline Female & Left eye PL +ve & 10 & $6 / 36$ & $6 / 24$ & $6 / 24$ & 12 & 12 & 12 \\
\hline Case 04 & Right eye PL +ve & 26 & $6 / 60$ & $6 / 36$ & $6 / 36$ & 20 & 18 & 18 \\
\hline \multirow{2}{*}{ Female } & Left eye PL + ve & 18 & $6 / 12$ & - & - & - & - & - \\
\hline & & & surgery not done & & & & & \\
\hline
\end{tabular}

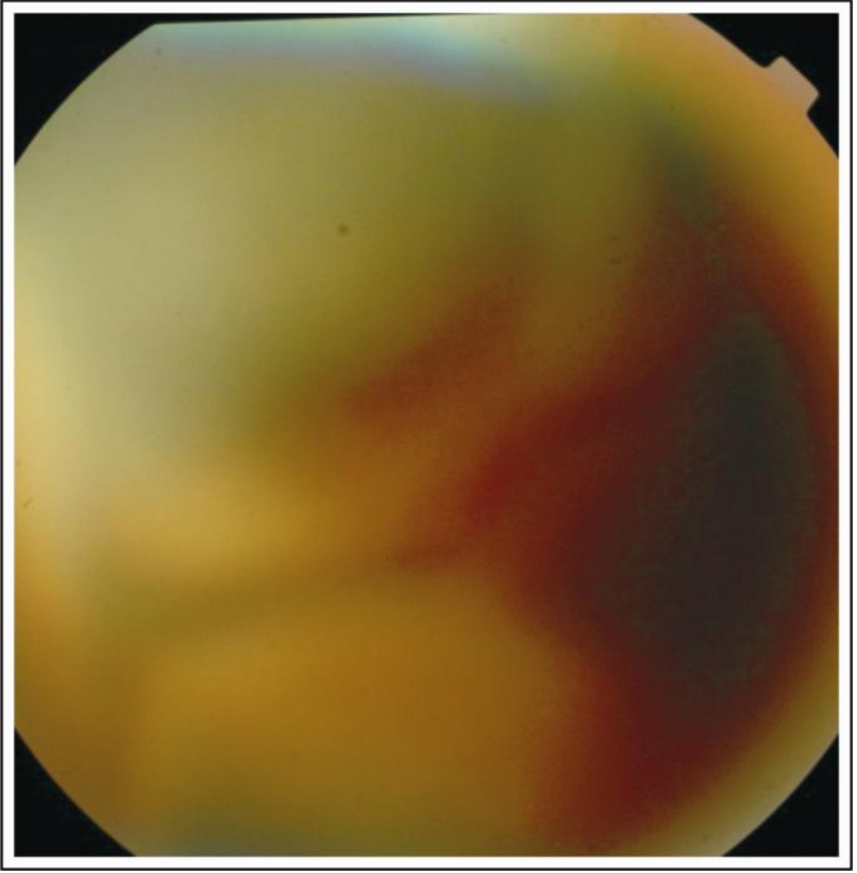

Fig. 1: Preoperative Fundsu picture case 1.

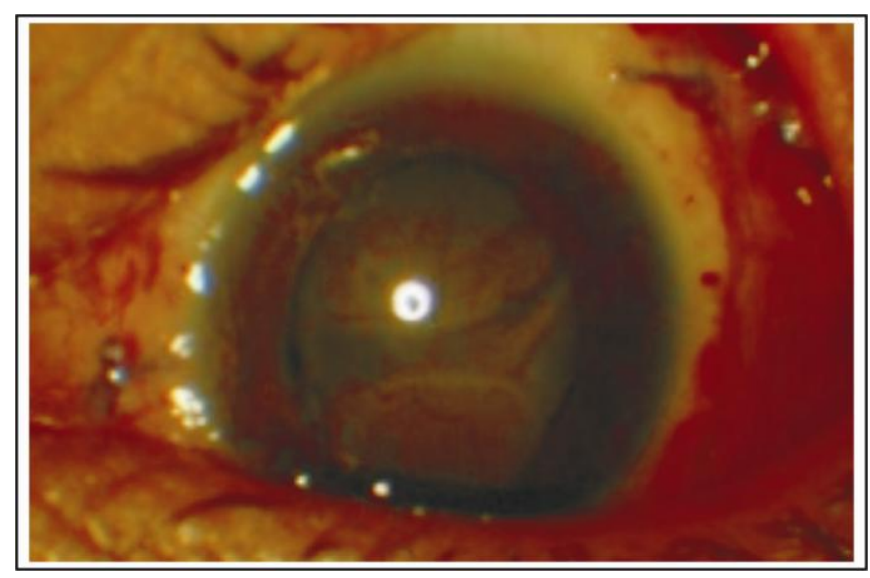

Fig. 2: First Postoperative day case 1.

\section{DISCUSSION}

There are multiple treatment modalities used for treatment of uveal effusion syndrome. Some ophthalmologists tried medical treatment for uveal

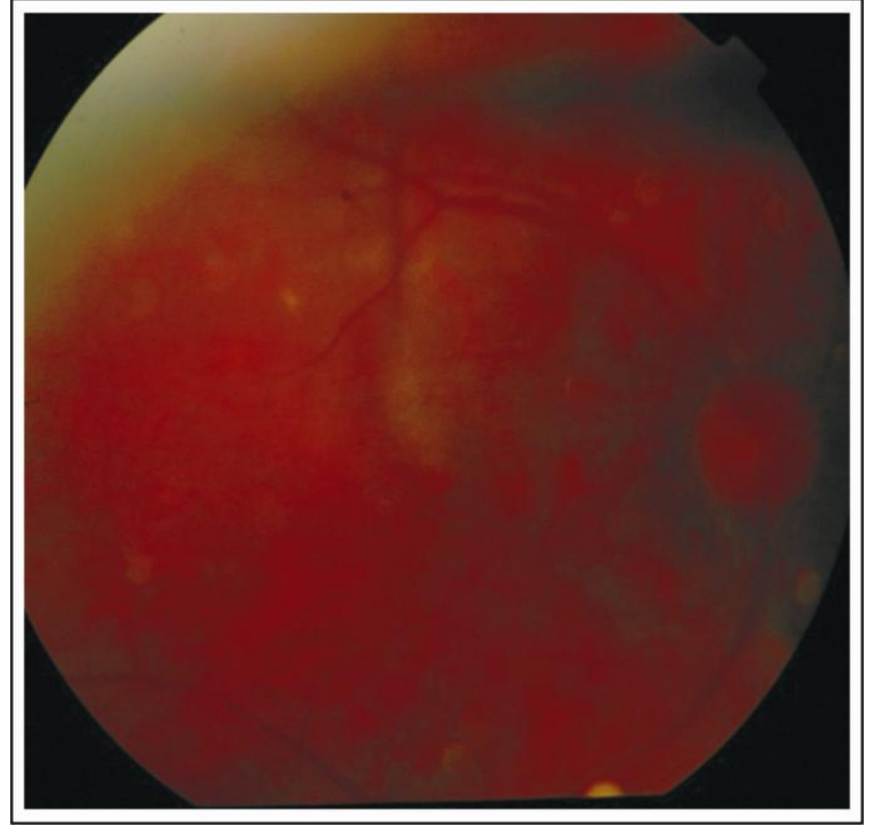

Fig. 3: Fundus picture -1 month Postoperatively case 1.

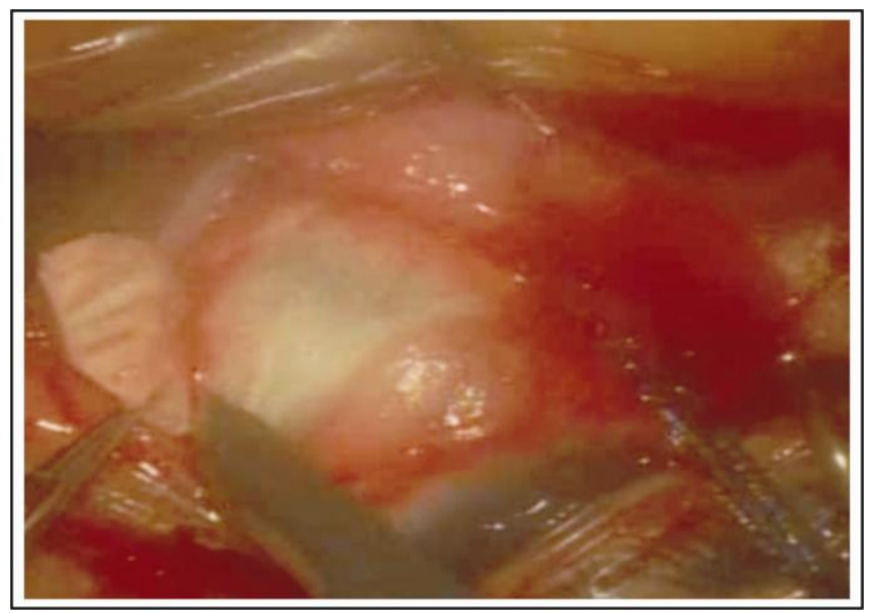

Fig. 4: One of the four Quadrant Sclerectomies.

effusion syndrome with oral steroid. Shield et al treated 104 eyes of uveal effusion syndrome with corticosteroids ${ }^{17}$ Bausili et al used $\mathrm{CO}_{2}$ laser ${ }^{18}$ and control was achieved in $95 \%$ cases in reports but 
according to others, surgical treatment was only effective option for treatment of idiopathic uveal effusion syndrome. We have found that surgical sclerectomies using partial thickness resection was very effective. We operated 6 eyes of 4 patients with idiopathic uveal effusion syndrome. Four Quadrant sclerectomies in 5 eyes and 2 quadrant sclerectomies in the $6^{\text {th }}$ eye was successful in attaining retinal reattachment. Following partial scleral resection all 6 eyes showed improvement in visual acuity. It was comparable with other studies done at other centers in London, USA and Bangladesh19,20,21.

Theories regarding pathogenesis of uveal effusion syndrome suggest that it is more common in nanophthalmic eyes ${ }^{11}$ where scleral pathology is congenital and abnormally thick sclera compresses vortex veins leading to impeded drainage. Similarly, thick sclera is also seen in patients suffering from glycogen storage disease, mucopolysaccharidosis (Hunter syndrome) where sclera is thick due to increased deposition of mucopolysaccharides. Gass attempted vortex vein decompression in a series of eyes and found that full thickness scleral incisions were effective in causing retinal re-attachment, supporting the hypothesis that it was primarily scleral thickening causing uveal effusion by a barrier effect to diffusion of extra vascular protein (albumin is major protein) out of sclera and obstruction of vortex veins. Abnormal scleral composition increases resistance to transcleral protein outflow, which in turn leads to accumulation of protein in extra vascular space of choroid causing higher colloidal osmotic pressure. Prostaglandin analogs and steroids have been tried with varying success. Surgical management also has various modifications and is usually successful although visual results are moderate. Choroidal effusion and non rhegmatogenous retinal detachment can be successfully treated in patients with uveal effusion syndrome by quadrantic partial thickness sclerectomies $^{20,21}$. The disappearance of serous fluid after partial thickness sclerectomies is consistent with hypothesis that abnormally thick sclera prevents outflow of protein and suggests that removal of excess extra vascular protein may be improved by reducing scleral thickness and resistance to fluid outflow.

Vortex vein decompression as a possible mechanism of uveal effusion in nanophthalmic eyes following glaucoma filtration surgery was first suggested by Schaffer in $1975^{22}$. In 1980, Brockhurst reported successful use of scleral thinning procedure with vortex vein decompression in treatment of nanophthalmic ciliochoroidal effusion ${ }^{23}$. Another case of bilateral UES was reported where the surgeon combined partial thickness scleral flaps with full thickness sclerectomies and reporting stable anatomic and visual results in the follow up period ${ }^{24,25}$.

Limitation of our study was the small number of cases. Large scale studies need to be conducted to further compare different surgical techniques. We also recommend that surgeons need to review the available literature before contemplating any specific surgical procedure for this rare condition.

\section{CONCLUSION}

Given that UES is a very rare disorder, we still do not have clear guidelines regarding its complete management. Partial thickness sclerectomy is an effective treatment option for patients of uveal effusion syndrome not responding to medical treatment.

\section{FINANCIAL DISCLOSURE}

There is no financial interest of authors.

\section{REFERENCES}

1. Von Graefe A. Zur diagnosis des beginnenden intraocularen krebses. Arch Ophthalmol. 1858; 4: 21802229.

2. Verhoeff FH, Waite JH. Separation of choroid with report of spontaneous case. Trans Am Ophthalmol Soc. 1925; 23: 120-139.

3. Schepens CL, Brockhurst RJ. Uveal effusion. I. Clinical picture. Arch Ophthalmol. 1963; 70: 189-210.

4. Gass JDM, Sulayman J. Idiopathic serous detachment of the choroid, ciliary body and retina (uveal effusion syndrome). Ophthalmology, 1982; 89: 1018-32.

5. Moses RA. Detachment of ciliary body - anatomical and physical considerations. Invest Ophthalmol. 1965; 4:935-41.

6. Davies EWG. Annular serous choroidal detachment. Mod. Probl. Ophthal. 1979; 20: 2-5.

7. Jackson TL, Hussain A, Salisbury J, Sherwood R, Sullivan PM, Marshall J. Transscleral albumin diffusion and suprachoroidal albumin concentration in uveal effusion syndrome. Retina. 2012; 32: 177-182.

8. Uyama M, Takahashi K, Kozaki J, Tagami N, Takada Y, Ohkuma H, Matsunaga H, Kimoto T, Nishimura T. Uveal effusion syndrome: clinical features, surgical treatment, histologic examination of the sclera, and pathophysiology. Ophthalmology, 2000; 107 (3): 441-9.

9. Trelstad RL, Silbermann NN, Brockhurst RJ. Nanophthalmic sclera: ultrastructural, histochemical, 
and biochemical observations. Arch Ophthalmol. 1982; 100: 1935-1938.

10. Okuda T, Higashide T, Wakabayashi Y. Fundus autofluorescence and spectral-domain optical coherence tomography findings of leopard spots in nanophthalmic uveal effusion syndrome. Graefes Arch Clin Exp Ophthalmol. 2010; 248: 1199-202.

11. Elagouz M, Stanescu-Segall D, Jackson TL. Uveal effusion syndrome. Surv Ophthalmol. 2010; 55: 134-145.

12. Andrijevic Derk B, Bencic G, Corluka V, Zoric Geber M, Vatavuk Z. Medical therapy for uveal effusion syndrome. Eye (Lond). 2014; 28 (8): 1028-31.

13. Brockhurst RJ. Vortex vein decompression for nanophthalmic uveal effusion. Arch Ophthalmol. 1980; 98: 1987-1990.

14. Yepez JB, Arevalo JF. Ex-Press shunt for choroidal fluid drainage in uveal effusion syndrome type2: a potentially novel technique. JAMA Ophthalmol. 2015; 133 (4): 470-1.

15. Kong M, Kim JH, Kim SJ, Kang SW. Full-thickness sclerotomy for uveal effusion syndrome. Korean J Ophthalmol. 2013; 27 (4): 294-298.

16. Jin W1, Xu Y2, Wang W1, Yang A1. Diagnosis and a minimum effective management for nanophthalmic uveal effusion syndrome. Indian J Ophthalmol. 2016; 64 (8): 593-4.

17. Shields CL, Roelofs K, Di Nicola M, Sioufi K, Mashayekhi A, Shields JA. Uveal effusion syndrome in 104 eyes: Response to corticosteroids-the 2017 Axel C.

\section{Author's Affiliation}

Dr. Muhammad Tariq Khan

Associate Professor (Ophthalmology)

Head of Vitreoretinal Department

Akhtar Saeed Trust Hospital, Lahore

Dr. Sidrah Riaz

Associate Professor (Ophthalmology)

Akhtar Saeed Trust Hospital, Lahore

Dr. Haroon Tayyab

Assistant Professor

King Edward Medical University, Lahore

Dr. Abdul Majeed Malik

Professor of Ophthalmology

Akhtar Saeed Medical \& Dental College, Lahore
Hansen Lecture. Indian J Ophthalmol. 2017; 65: 1093104.

18. Bausaili MM, Raja H, Kotowski J, Nadal j, Salomoa Dr, Keenum D, et al. Use of fiberoptic guided Co2 in treatment of uveal effusion. Retina case brief Rep. 2017; 11: 191-4.

19. Wang BZ, Clark B, Mckelvie P, Matthews BJ, Buttery RG, Chandra A, et al. Four quadrant sclerectomies for uveal effusion syndrome. Eye (Lond), 2015; 29: 588-9.

20. Ozgonul C, Dedania VS, Cohen SR, Besirli CG. Scleral surgery for uveal effusion. Retina, 2017; 37: 1977-83.

21. Shah PR, Yohendran J, Hunyor AP, Grigg JR, McCluskey PJ. Uveal effusion: Clinical features, management, and visual outcomes in a retrospective case series. J Glaucoma, 2016; 25: e329-35.

22. Calhoun FP. The management of Glaucoma in nanophthalmos. Trans. Am. Ophthalmol. Soc. 1975, 73: 98-122.

23. Abell RG1, Kerr NM, Vote BJ. Bilateral nanophthalmic uveal effusion syndrome: clinical presentation and surgical management. Retin Cases Brief Rep. 2013 Fall; 7 (4): 386-90.

24. Schneiderman TE, Johnson MW. A new Approach to the surgical management of idiopathic uveal effusion syndrome Am J Ophthalmol. 1997; 123: 262-263.

25. Elagouz M, Stanescu-Segall D, Jackson TL. Uveal Effusion Syndrome. Surv Ophthalmol. 2010; 55: 134-145.

\section{Author's Contribution}

Dr. Muhammad Tariq Khan

Study design, Manuscript drafting, Data collection, Data analysis, performed surgeries.

Dr. Sidrah Riaz

Data collection, Data analysis, article writing, Manuscript review.

Dr. Haroon Tayyab

Data collection, Manuscript drafting, Manuscript review.

Dr Majeed Malik

Data analysis, Manuscript review. 